\title{
The New Horizons Mission to Pluto-Charon and the Kuiper Belt
}

\author{
G. Leonard Tyler \\ Stanford University, Packard 331, 350 Serra Mall, Stanford, CA \\ 94305-9515,USA, len.tyler@stanford.edu
}

\section{S. Alan Stern}

Southwest Research Institute, Suite 400, 1050 Walnut Street, Boulder CO 80302, USA, astern@swri.edu

\author{
Harold A. Weaver \\ Johns Hopkins University/Applied Physics Laboratory, 11100 Johns \\ Hopkins Road, Laurel, MD 20723-6099, USA, hal.weaver@jhuapl.edu
}

\begin{abstract}
The New Horizons Mission to Pluto-Charon and the Kuiper Belt is under development for launch in 2006. The experiment complement of eight sensors addresses fundamental issues of density, composition, albedo, geology, and atmospheric state, chemistry, and escape.
\end{abstract}

The New Horizons (NH) Mission, currently in the middle stages of development by Southwest Research Inst. and Johns Hopkins University Applied Physics Lab., is designed to provide the first detailed, spacecraft observations of the Pluto-Charon system and smaller Kuiper-Belt Objects (KBOs) beyond. An $\approx 465 \mathrm{~kg}$ spacecraft is to be launched on a Jupiter gravity-assist trajectory in January 2006, to arrive at the Pluto-Charon system by 2016, after which it will continue for several more years to the Kuiper Belt. The trajectory includes scientifically useful, moderate-range, $\approx 38 R_{\mathrm{J}}$, observations of Jupiter followed by an $\approx 12 \mathrm{~km} / \mathrm{s}$ 'fly-through' of the Pluto-Charon system with a spacecraft closest approach to Pluto's center of mass of $\approx 11,000 \mathrm{~km}$, passing through the orbital plane of Charon. The encounter geometry includes Sun and Earth occultations by both Pluto and Charon. Subsequent to Pluto encounter, the spacecraft will maneuver to encounter at least one, and possibly two, KBOs between 2019 and 2026.

The instrument complement, Table 1, provides for visible and IR spectral mapping, UV spectroscopy, solar-UV and Earth-radio occultations, in situ sensing of system plasma, and radio-tracking determination of Pluto and Charon masses. Planned observations will characterize Pluto's suspected complex geology on scales less than $1 \mathrm{~km}$, map surface composition, determine the volatiles and structure of the neutral atmosphere, determine the rate of atmospheric escape from the system, and characterize the possible exchange of volatiles between Pluto and Charon. A long focal length camera is included for the purpose of providing observations of the Pluto system for six months prior to closest approach. This will permit study of Pluto and Charon over many rotations, and provide context for the close encounter. Imaging sensitivity is such that the dark 
Table 1. New Horizons Payload Summary. ( 'NA' = narrow angle, 'Pan' = pan-chromatic, 'Spect' = spectrometer.)

\begin{tabular}{lll}
\hline \hline Investigation & Type & Sensor Characteristics \\
\hline RALPH & Visible mapping imager & Pan- and 4-color CCD \\
LEISA & Near-IR imaging spect & $1.24-2.5 ; 2.1-2.25 \mu \mathrm{m}$ \\
ALICE & UV imaging spect & $520-1870 \AA$ \\
REX & Radio occultation $T(p[z])$ & $\Delta p=0.01 \mathrm{~Pa}, \Delta T_{\text {sur }}=1 \mathrm{~K}$ \\
& Microwave radiometry & $4.2 \mathrm{~cm}-\lambda, \Delta T=0.1 \mathrm{~K}$ \\
& Body mass & Two-way radiometric tracking \\
SWAP & Plasma spect & To $6.5 \mathrm{keV}$ \\
PEPSSI & Hi-energy particles & Ions: $1-3000, \mathrm{e}^{-}: 25-700 \mathrm{keV}$ \\
LORRI & Long focal length imager & $\mathrm{NA}, \mathrm{Pan} \mathrm{CCD}, 5 \mu \mathrm{rad} / \mathrm{pixel}$ \\
SDC & Student dust counter & area $0.25 \mathrm{~m}^{2}$, mass $>10^{-15} \mathrm{~kg}$ \\
\hline \hline
\end{tabular}

side of Pluto-otherwise not seen at high resolution - can be imaged in 'Charonshine' as NH departs the system. The same instrument set will be effective in reconnaissance of KBOs, with primary emphasis on surface characteristics.

The Student Dust Counter is provided by joint agreement between $\mathrm{NH}$ Project and the University of Colorado. To be operated by a succession of undergraduate and graduate students at $\mathrm{CU}$, this instrument will provide the first in situ determination of the dust distribution between Earth and the outer solar system, providing a basis for testing Earth-based inferences and theories of dust distribution.

The mission design satisfies all NASA (2001) and PKB Science Definition Team (Lunine et al., 1995) Group I objectives, and virtually all Group II and III objectives (Stern \& Cheng, 2002).

The choice of $\mathrm{KB}$ targets is to be made between now and the approach to Pluto-Charon, based on new KB surveys over the next decade. On the basis of the known KBO's, the current rate of their discovery, and the capabilities of the NH spacecraft, Monte Carlo simulations indicate NH will be able to reach one or two KBO's exceeding $35 \mathrm{~km}$ in diameter, depending upon the orbits of the target bodies and the performance of the $\mathrm{NH}$ navigation and propulsion systems. The instrument complement can readily address questions of KBO albedos, masses, and densities, which will support studies of bulk composition and state. Similarly, it will be possible to study surface geology, surface composition and its variation, and to search for tenuous atmospheres.

\section{References}

Lunine, J.I., et al., 1995. Report of the Pluto-Kuiper Express Science Definition Team. (NASA, unpublished)

NASA, 2001. Pluto Kuiper Belt Mission Announcement of Opportunity. AO-01-OSS-01

Stern, S. A., and A. Cheng, 2002. NASA Plans Pluto-Kuiper Belt Mission. EoS, 83, 101 